

\title{
Magnetization dynamics in nanostructures with weak/strong anisotropy
}

A. M. H. de Andrade, M. A. Corrêa, A. D. C. Viegas, F. Bohn, and R. L. Sommer

Citation: Journal of Applied Physics 115, 103908 (2014); doi: 10.1063/1.4868157

View online: http://dx.doi.org/10.1063/1.4868157

View Table of Contents: http://scitation.aip.org/content/aip/journal/jap/115/10?ver=pdfcov

Published by the AIP Publishing

\section{Articles you may be interested in}

Fabrication of dot pattern using magnetic phase change on Pt ion-implanted L10 FePtRh film with high magnetocrystalline anisotropy

J. Appl. Phys. 115, 17B721 (2014); 10.1063/1.4864744

Tunable magnetic anisotropy in permalloy thin films grown on holographic relief gratings

Appl. Phys. Lett. 104, 082408 (2014); 10.1063/1.4867162

The low-frequency alternative-current magnetic susceptibility and electrical properties of $\mathrm{Si}(100) / \mathrm{Fe} 40 \mathrm{Pd} 40 \mathrm{~B} 20(\mathrm{X} \AA) / \mathrm{ZnO}(500 \AA)$ and $\mathrm{Si}(100) / \mathrm{ZnO}(500 \AA) / \mathrm{Fe} 40 \mathrm{Pd} 40 \mathrm{~B} 20(\mathrm{Y} \AA)$ systems J. Appl. Phys. 113, 17B303 (2013); 10.1063/1.4794985

Magnetization reversal in graded anisotropy Co/Pt multilayers: A first order reversal curve study J. Appl. Phys. 112, 123914 (2012); 10.1063/1.4770487

Edge atoms effects on the perpendicular anisotropy of ultrathin magnetic layers Appl. Phys. Lett. 101, 132407 (2012); 10.1063/1.4754621

\section{A.P $\left.\right|_{\text {Applied Physics }} ^{\text {Journal of }}$}

Journal of Applied Physics is pleased to announce André Anders as its new Editor-in-Chief 


\title{
Magnetization dynamics in nanostructures with weak/strong anisotropy
}

\author{
A. M. H. de Andrade, ${ }^{1}$ M. A. Corrêa, ${ }^{2}$ A. D. C. Viegas, ${ }^{3}$ F. Bohn, ${ }^{4}$ and R. L. Sommer, \\ ${ }^{1}$ Instituto de Física, Universidade Federal do Rio Grande do Sul, 91501-970 Porto Alegre, RS, Brazil \\ ${ }^{2}$ Departamento de Física Teórica e Experimental, Universidade Federal do Rio Grande do Norte, \\ 59078-900 Natal, RN, Brazil \\ ${ }^{3}$ Departamento de Física, Universidade Federal de Santa Catarina, 88010-970 Florianópolis, SC, Brazil \\ ${ }^{4}$ Escola de Ciências e Tecnologia, Universidade Federal do Rio Grande do Norte, 59078-900 Natal, RN, \\ Brazil \\ ${ }^{5}$ Centro Brasileiro de Pesquisas Físicas, Rua Dr. Xavier Sigaud 150, Urca, 22290-180 Rio de Janeiro, RJ, \\ Brazil
}

(Received 4 December 2013; accepted 27 February 2014; published online 13 March 2014)

\begin{abstract}
We investigate the high-frequency response of magnetization dynamics through magnetoimpedance (MI) effect in Permalloy-based multilayered thin films produced with two different non-magnetic metallic spacers: $\mathrm{Cu}$ and $\mathrm{Ag}$. Due to the nature of the spacer materials, we are able to play with magnetic properties and to study both systems with weak/strong magnetic anisotropy. We verify very rich features in the magnetoimpedance behavior and high magnetoimpedance ratios, with values above $200 \%$. We compare the MI results obtained in multilayered thin films with distinct spacers and number of bilayers, and discuss them in terms of the different mechanisms that govern the MI changes observed at distinct frequency ranges, intensity of the magnetic anisotropy, alignment between $d c$ magnetic field and anisotropy direction. Besides, by considering a theoretical approach that takes into account two single models together and calculate the transverse magnetic permeability and the MI effect, we support our interpretation via numerical calculations modeling the effect of weak/strong magnetic anisotropy on the MI response. Thus, we confirm that these features are very important for the use of multilayered films in sensor applications and, both the frequency and field response can be tailored to fulfill the requirements of a given device. (C) 2014 AIP Publishing LLC. [http://dx.doi.org/10.1063/1.4868157]
\end{abstract}

\section{INTRODUCTION}

The magnetization dynamics in films and nanostructures is a fundamental issue for current and emerging technologies and devices as MRAMs, ${ }^{1}$ spin torque nano-oscillators, ${ }^{2,3}$ and magnetic read heads. ${ }^{4}$ In this sense, magnetoimpedance (MI) is one of the more versatile tools to perform the characterization of the magnetic materials and nanostructures composing these devices, revealing their magnetic properties at different frequency ranges and magnetic field values, even at nonsaturated magnetization regimes.

The MI effect corresponds to the change of the complex electric impedance $Z=R+i X$ of a ferromagnetic conductor submitted to a static external magnetic field $H$. Thus, in a typical MI experiment, besides the external field, the sample is also submitted to an alternate magnetic field associated with the probe electric current $I_{a c}=I_{o} \exp (i 2 \pi f t), f$ being the current frequency.

The overall effect of these magnetic fields is to induce strong modifications of the effective magnetic permeability, a factor that is relevant to determine the fields and current distributions within the sample. ${ }^{5}$ It is observed that in a soft magnetic material, the magnetic permeability can change several orders of magnitude when a rather small field is applied, leading to strong variations of the sample's impedance. ${ }^{6}$

Traditionally, the changes of magnetic permeability and consequently impedance with magnetic fields at different

${ }^{\text {a)} E l e c t r o n i c ~ a d d r e s s: ~ s o m m e r @ c b p f . b r ~}$ frequency ranges are interpreted considering three distinct mechanisms: magnetoinductive effect, skin effect, and ferromagnetic resonance (FMR). It is well-known that at intermediate and high frequencies, above hundreds of $\mathrm{MHz}$, the skin depth and FMR (by skin effect) command the MI effect through variations of the transverse magnetic permeability, which has a strong dependence with the magnetic properties of the sample. ${ }^{7}$ However, even from this traditional point of view, the precise limits between the frequency ranges are strongly dependent on magnetic anisotropy and, particularly, on the dimensions and geometry of the sample. Therefore, the control of these properties is a key issue on having a good MI response in a given material. Besides, by choosing an appropriate material and structure, it is possible to tailor desired impedance responses, depending on the given technological application.

The higher MI ratio is usually observed in soft magnetic materials with well defined magnetic anisotropy and low electrical resistance. ${ }^{6,8}$ Since the discovery of the effect, MI has been studied in a wide variety of systems, e. g., sheets, ${ }^{9}$ wires, ${ }^{10-18}$ ribbons, ${ }^{19-24}$ and thin films. ${ }^{25-39}$ In the particular case of films, an increasing interest has been devoted to the effect in single layered, ${ }^{25,26}$ multilayered, ${ }^{27-32}$ and structured multilayered thin films, ${ }^{30,31,33-39}$ since these nanostructures are suitable for applications in integrated devices (magnetic field sensor + integrated circuit $^{40}$ ), in bio $^{41}$ and geo-sensors, ${ }^{42}$ etc.

In previous works reported by our group,,$^{30,31,37-39}$ the use of multilayered films allowed us to control the magnetic 
anisotropy and stored stress in ferromagnetic layers, as well as to tailor the $d c$ resistance and, consequently, the MI effect.

In this paper, we report an experimental investigation of the high-frequency response of magnetization dynamics through magnetoimpedance effect in Permalloy-based multilayered thin films produced with two different non-magnetic metallic spacers: $\mathrm{Cu}$ and $\mathrm{Ag}$. By playing with the nature of the spacers, we are able to produce samples with distinct effective magnetic anisotropies and experimentally confirm that magnetic anisotropy has a fundamental role in the magnetoimpedance effect behavior. We show that the magnetoimpedance in these systems presents very rich features (single, double and multiple peak structures) and high MI ratios, with values above $200 \%$, which bring new horizons for application and devices design. In particular, we compare the MI results obtained in multilayered thin films with distinct spacers and number of bilayers, and discuss them in terms of the different mechanisms that govern the MI changes observed at distinct frequency ranges, intensity of the magnetic anisotropy, alignment between $d c$ magnetic field and anisotropy direction. By considering a theoretical approach that takes into account two single models together and calculate the transverse magnetic permeability and the MI effect, we support our interpretation via numerical calculations modeling the effect of weak/strong magnetic anisotropy on the MI response. Thus, we confirm that these features are very important for the use of multilayered films in sensor applications and, both the frequency and field response can be tailored to fulfill the requirements of a given device.

\section{EXPERIMENT}

In this work, we study multilayered thin films of $(\mathrm{Py} / \mathrm{NM}) \times n$, where Py is the well-known Permalloy with nominal composition $\mathrm{Ni}_{81} \mathrm{Fe}_{19}$, $\mathrm{NM}$ is the spacer material $\mathrm{Ag}$ or $\mathrm{Cu}$, and $n$ is the number of bilayers Py/NM. In particular, we consider 15,50 , and 100 as values of $n$, while $t_{P y}=10 \mathrm{~nm}$ and $t_{N M}=2.5 \mathrm{~nm}$ are the thicknesses of the Py magnetic and $\mathrm{Ag}$ or $\mathrm{Cu}$ non-magnetic metallic layers, respectively. The complete list of the studied samples is presented in Table I.

The films are grown by magnetron sputtering onto glass substrates with dimensions of $50 \mathrm{~mm} \times 24 \mathrm{~mm}$, covered by a Ta buffer layer. The deposition is carried out with the following deposition parameters: base pressure of $5.0 \times 10^{-7}$ Torr, Ar pressure during deposition of $5.2 \mathrm{mTorr}$ and $20 \mathrm{sccm} \mathrm{Ar}$ flow rate, $65 \mathrm{~W}$ RF power supply for the Permalloy deposition, and a $d c$ current of $10 \mathrm{~mA}$ and $25 \mathrm{~mA}$ for the $\mathrm{Ag}$ and $\mathrm{Cu}$ growths, respectively. Using these parameters, the deposition

TABLE I. Set of samples studied in this work.

\begin{tabular}{lcrc}
\hline \hline Label & Spacer & $n$ & $\mathrm{t}_{P y} / \mathrm{t}_{N M}(\mathrm{~nm})$ \\
\hline PyCu $_{15}$ & $\mathrm{Cu}$ & 15 & $10 / 2.5$ \\
$\mathrm{PyCu}_{50}$ & $\mathrm{Cu}$ & 50 & $10 / 2.5$ \\
$\mathrm{PyCu}_{100}$ & $\mathrm{Cu}$ & 100 & $10 / 2.5$ \\
$\operatorname{PyAg}_{15}$ & $\mathrm{Ag}$ & 50 & $10 / 2.5$ \\
$\operatorname{PyAg}_{50}$ & $\mathrm{Ag}$ & 100 & $10 / 2.5$ \\
$\operatorname{PyAg}_{100}$ & $\mathrm{Ag}$ & & $10 / 2.5$ \\
\hline \hline
\end{tabular}

rates are $0.27 \mathrm{~nm} / \mathrm{s}$ for Permalloy, $0.15 \mathrm{~nm} / \mathrm{s}$ for $\mathrm{Ag}$, and $0.16 \mathrm{~nm} / \mathrm{s}$ for $\mathrm{Cu}$. The deposition is done with the substrate moving at constant speed through the plasma to improve film uniformity. During the deposition process, the substrate is submitted to a 100 Oe axial stray magnetic field from the sputtering gun. This stray field, associated with the motion of the substrate, induces an in-plane uniaxial anisotropy axis in the multilayered films during the growth of the samples. The easy magnetization axis is observed to be perpendicular to the direction of motion of the substrates. Once the film is produced, samples with dimensions of $10 \mathrm{~mm} \times 4 \mathrm{~mm}$ are cut from the matrix film, with the main axis oriented along (labeled HA) and perpendicular (EA) to the direction of motion of the substrate, as shown in Fig. 1.

The structural properties of the multilayered thin films are obtained by X-ray diffractometry (XRD), not shown here. Low-angle XRD measurements are performed to verify the interface behavior and chemical modulation of the sample. High-angle XRD measurements confirm the polycrystalline structural character, with (111) preferential growth for all layers.

The quasi-static magnetic properties are obtained through magnetization curves measured using a vibrating sample magnetometer, at room temperature, with maximum magnetic field of \pm 150 Oe, applied along the main axis of the EA and HA samples.

The magnetoimpedance effect is measured using an HP4396B spectrum-impedance-network analyzer equipped with an HP43961A impedance measuring fixture. In this case, a stripline geometry is used, with the sample playing the role of the central conductor in the sample holder, which is separated from the ground plane by the glass substrate, as detailed in Fig. 2. The electric contacts between the sample and the sample holder are made with $24 \mathrm{~h}$ cured low resistance silver paint. To avoid propagative effects and acquire just the sample contribution to MI, the analyzer is calibrated at the end of the connection cable by performing open, short, and load $(50 \Omega)$ measurements using reference standards. The probe current is fed directly to one side of the sample,

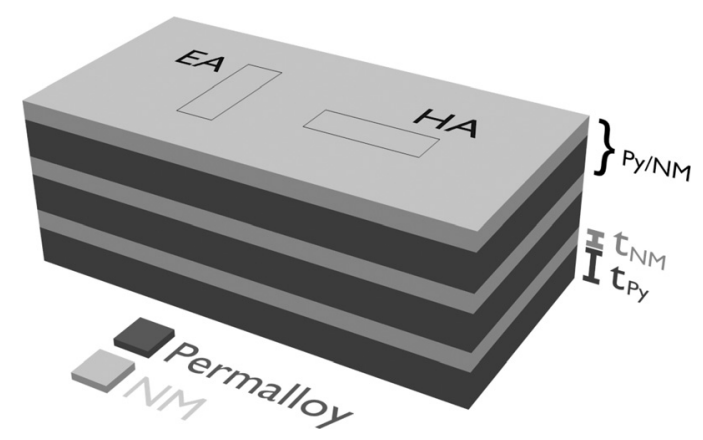

FIG. 1. Schematic diagram of the multilayered films prepared in this work, indicating the cut orientation of the samples employed in the experiments. During the deposition, the matrix substrate is submitted to a magnetic field, not shown here. Thus, according the relative orientation between the main axis of the cut sample and magnetic field, the cut samples are named as HA and EA, where the first has the main axis oriented along the direction of the motion of the substrate during deposition and the second one perpendicularly to the first. Here, Py/NM corresponds to a single bilayer, and $t_{P y}$ and $t_{N M}$ represent the Py magnetic and non-magnetic metallic layer thicknesses, respectively. 

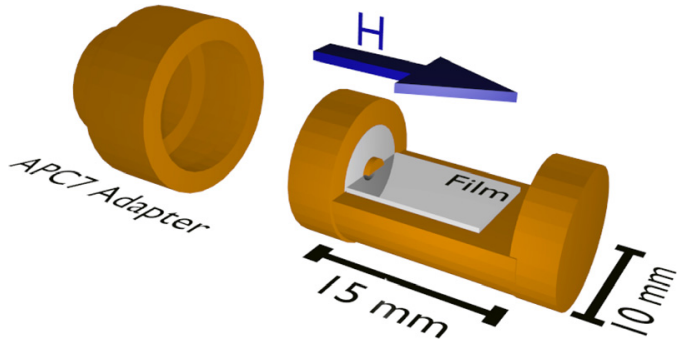

FIG. 2. Stripline geometry sample holder, with the sample, used to perform the impedance measurements.

while the other side is in short circuit with the ground plane. In all cases, the $a c$ current and $d c$ magnetic field are applied along the length of the sample. MI measurements are taken over a wide frequency range between $100 \mathrm{kHz}$ and $1.8 \mathrm{GHz}$, with maximum applied magnetic fields of \pm 150 Oe. For all measurements, while the external magnetic field is swept, a $0 \mathrm{dBm}(1 \mathrm{~mW})$ constant power is applied to the sample, with ac current amplitude set to $4.4 \mathrm{~mA}_{r m s}$, characterizing a linear regime of driving signal. Measurements with different values of the $a c$ current amplitude were performed, but they exhibited similar results, with a slight improvement of the signal/ noise ratio for the higher currents. Thus, in the measurement, at a given field value, the frequency sweep is made and the real and imaginary parts of line impedance are simultaneously acquired. For further information on the whole procedure, we suggest Refs. 26 and 37. The curves are known to exhibit hysteretic behavior, associated with the coercive field. However, in order to clarify the general behavior, only curves depicting the field going from negative to positive values are presented.

The MI ratio is quantitatively associated with the wellknown magnetoimpedance ratio $M I \%$, defined as ${ }^{25}$

$$
M I \%=\frac{\left[Z(H)-Z\left(H_{\max }\right)\right]}{Z\left(H_{\max }\right)} \times 100,
$$

where $Z(H)$ is the impedance value at a given field $H$ and $Z\left(H_{\text {max }}\right)$ is the impedance at the maximum applied magnetic field $H_{\text {max }}$, where the magnetic state of the sample is saturated. Finally, $\mathrm{MI}_{\max }$ is defined as the maximum $M I \%$ value for a given frequency.

\section{RESULTS AND DISCUSSION}

In this section, we present the experimental results of the $M$ vs. $H$ curves, $Z$ vs. $H$, and $\mathrm{MI}_{\max }$ vs. $f$, for $\mathrm{Py} / \mathrm{Cu}$ and $\mathrm{Py} / \mathrm{Ag}$ set of multilayered films produced in this work, considering the EA and HA cuts. Besides, we discuss the magnetoimpedance results in terms of the mechanisms that govern the MI changes, intensity of the magnetic anisotropy, alignment between $d c$ magnetic field and anisotropy direction, as well as we support our interpretation via numerical calculations modeling the effect of weak/strong magnetic anisotropy on the MI response.

\section{A. Magnetic behavior}

Magnetic characterization of the sets of multilayered films is obtained through magnetization curves. Figure 3 shows the quasi-static magnetization curves for all films, obtained with the magnetic field applied along the main axis of the EA and HA cuts.

For both set of films, the angular dependence of the magnetization curves indicates the existence of an uniaxial in-plane magnetic anisotropy induced during film growth. In general, irrespective of the anisotropy, the hysteresis loops at low fields for the HA cuts indicate that some stress is
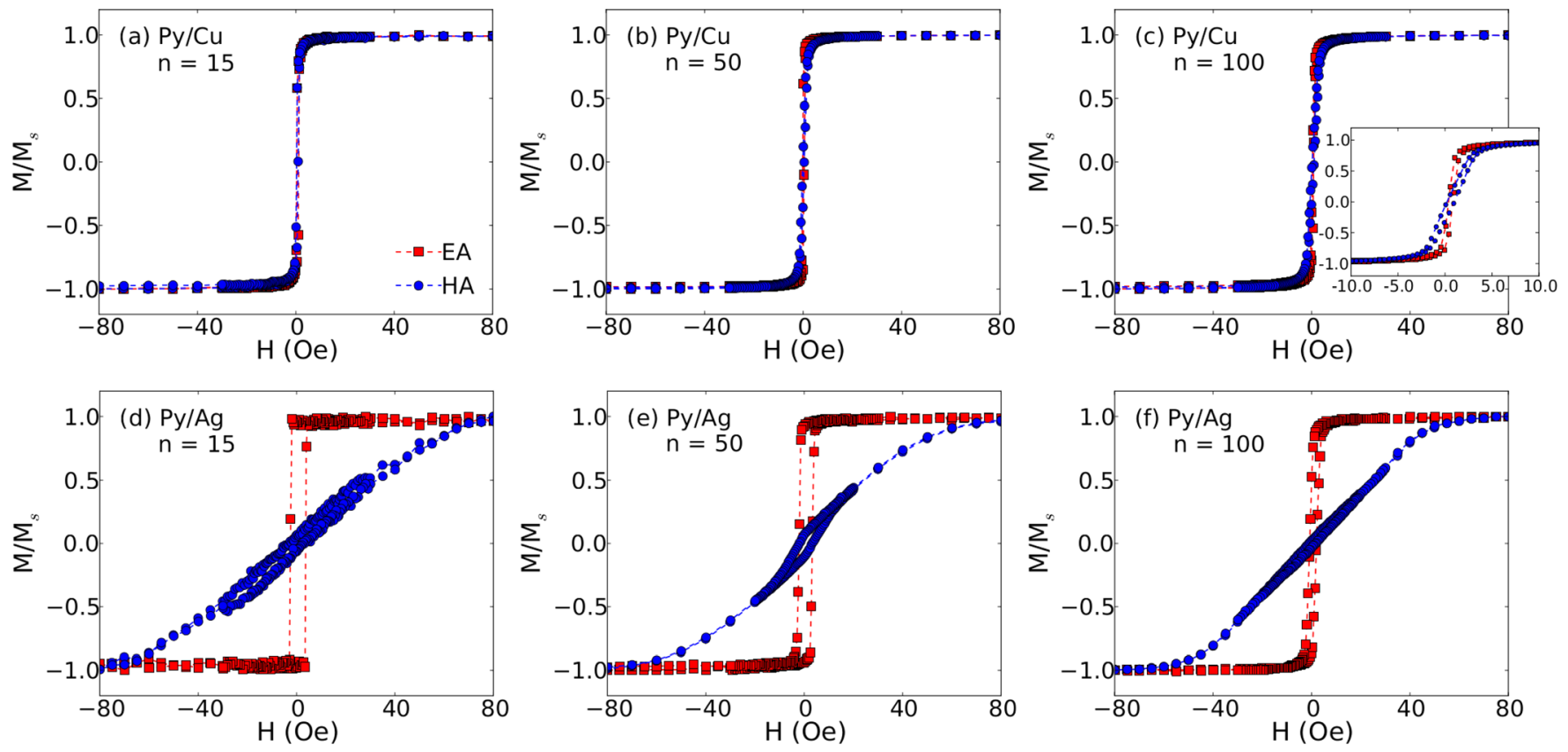

FIG. 3. Normalized magnetization curves for the Py/Cu and Py/Ag set of multilayered films, obtained for the EA and HA cuts. The magnetization curves are obtained between \pm 150 Oe, however they are presented between \pm 80 Oe to make the visualization clear. The weak and strong induced magnetic anisotropies are observed for the Py/Cu and Py/Ag sets, respectively. The inset in (c) emphasizes the weak magnetic anisotropy in the Py/Cu multilayered films. 
associated with pinning centers and domain structure. Besides, it is noticeable that the anisotropy induction in the $\mathrm{Py} / \mathrm{Cu}$ multilayered films is weaker than that observed for the Py/Ag ones, a behavior consistent with results previously reported for multilayered films with $\mathrm{Cu}$ and $\mathrm{Ag}$ employed as metallic spacer materials. ${ }^{31,32}$

In particular, for the $\mathrm{Py} / \mathrm{Cu}$ multilayered films, presented in Figs. 3(a)-3(c), due to the weak anisotropic magnetic behavior, the saturation field $H_{s}$ values are close for both cuts, being $\sim 1.0$ Oe for EA and $\sim 2.5$ Oe for HA, independently on the number of bilayers. On the other hand, for the Py/Ag films, shown in Figs. 3(d)-3(f), the $H_{s}$ values are between $55 \mathrm{Oe}$ and $75 \mathrm{Oe}$ for the HA cuts and $\sim 5 \mathrm{Oe}$ for the EA ones with different number of bilayers. Besides, a small reduction of the magnetic anisotropy is observed with the increase in the number of bilayers, a fact evidenced by the decrease in the coercive field of EA samples, and related to orange peel and similar effects.
It is well known that the intensity of the effective magnetic anisotropy and the alignment between $a c$ and $d c$ fields with the easy and hard magnetization axes are responsible for the behavior of the magnetization dynamics and the peaks' structure in the MI effect. ${ }^{25}$ Thus, the magnetic behavior observed for the $\mathrm{Py} / \mathrm{Cu}$ and $\mathrm{Py} / \mathrm{Ag}$ multilayered films is reflected in the magnetoimpedance results.

\section{B. High-frequency response of magnetization dynamics}

\section{Magnetoimpedance results}

Here, we present the high-frequency response of magnetization dynamics, investigated through the MI measurements. Figures 4 and 5 show the general view of the MI effect results for $\mathrm{Py} / \mathrm{Cu}$ and $\mathrm{Py} / \mathrm{Ag}$ multilayered films, respectively. For both, the results are plotted for the films with ( $\mathrm{a}$ and b) 15, (c and d) 50, and (e and f) 100 bilayers.
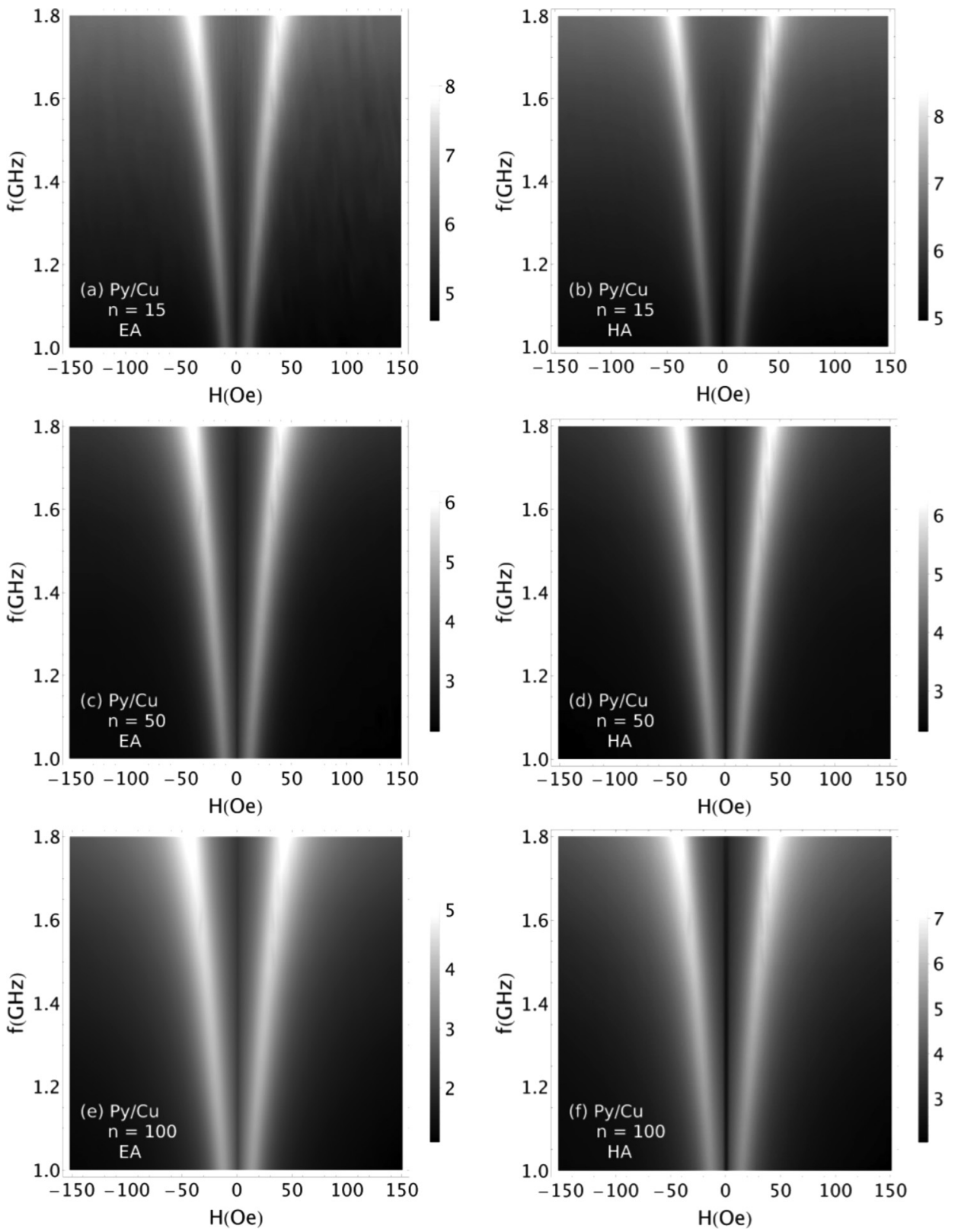

FIG. 4. Two-dimensional plot of the impedance $Z$ as a function of the external magnetic filed $H$ and frequency $f$ for the (a) and (b) $\mathrm{PyCu}_{15}$; (c) and (d) $\mathrm{PyCu}_{50}$; and (e) and (f) $\mathrm{PyCu}_{100}$ samples. Left: $Z$ behavior for EA cuts of the multilayered films. Right: $Z$ behavior for HA cuts. The presented frequency range is between $1.0 \mathrm{GHz}$ and $1.8 \mathrm{GHz}$ and the external magnetic field is between $\pm 150 \mathrm{Oe}$. 

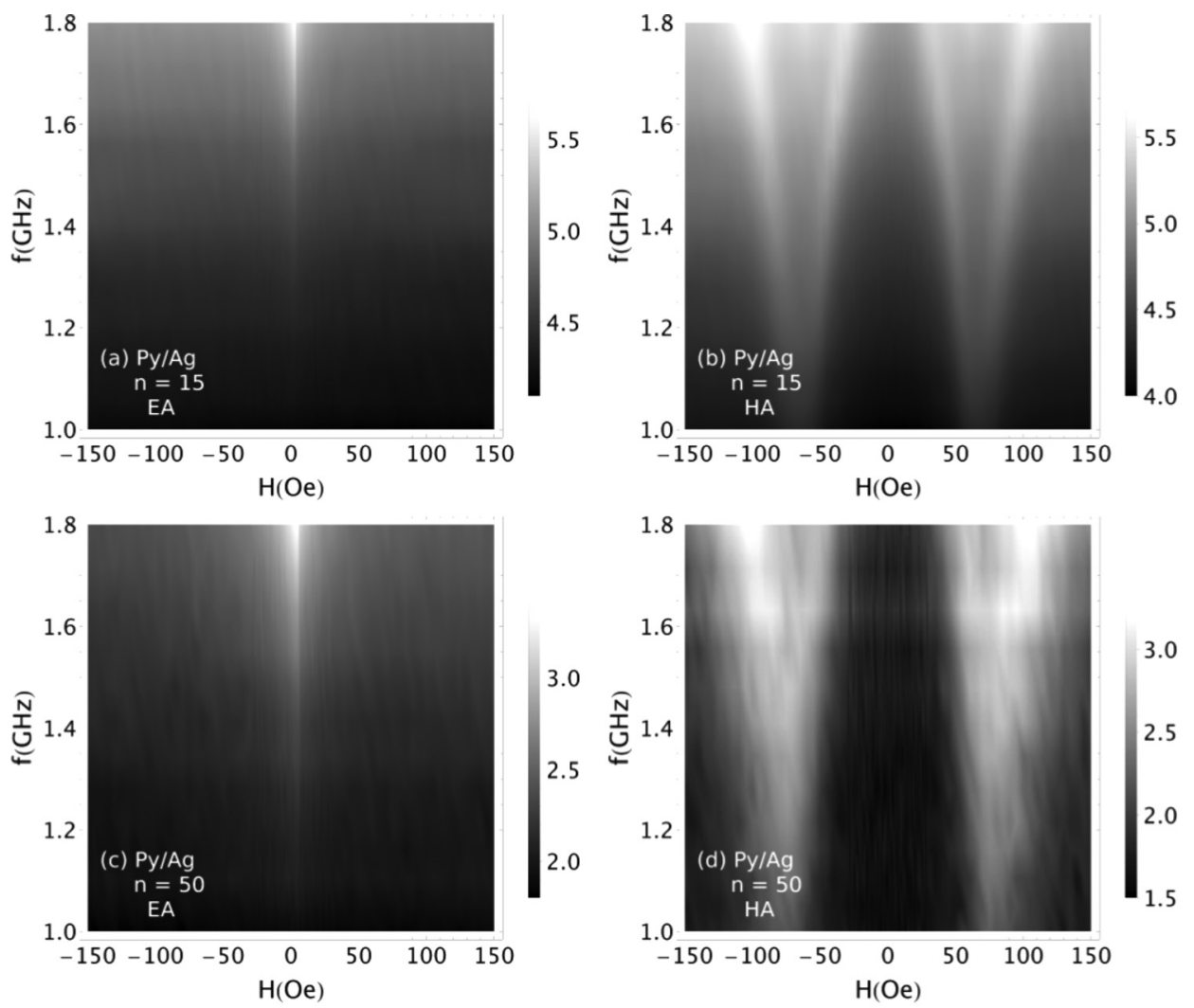

FIG. 5. Two-dimensional plot of the impedance $Z$ as a function of the external magnetic filed $H$ and frequency $f$, similar to the one presented in Fig. 4; (a) and (b) $\mathrm{PyAg}_{15}$, (c) and (d) $\mathrm{PyAg}_{50}$; and (e) and (f) $\mathrm{PyAg}_{100}$ samples. Left: $Z$ behavior for EA cuts of the multilayered films. Right: $Z$ behavior for HA cuts of the multilayer.


By considering this two-dimensional plot, it is possible to observe the behavior of the impedance $Z$ simultaneously as a function of the external magnetic field $H$ and frequency $f$.

For the $\mathrm{Py} / \mathrm{Cu}$ multilayered films, at low frequencies (not shown), the peak's configuration evolves from a single to a double peak structure. At moderate frequencies, around $0.5 \mathrm{GHz}$, the $Z$ peak's position is close to the $H_{s}$ values indicated in Sec. III A. This feature reflects the fact that the skin effect is the main responsible for the MI variations. Beyond $0.5 \mathrm{GHz}$, the $Z$ peak position in accordance with $H$, as $f$ increases, reflects the fact that the strong skin effect and a small contribution of the FMR effect become the main mechanisms responsible for the MI variations.

The contribution of the FMR effect to $Z$ was verified using the method described by Barandiarán et al., ${ }^{43}$ and previously employed by our group in Ref. 31 . This contribution at moderate frequency ranges may be associated with the presence of random local anisotropies induced by stress. Therewith, in this set of films, the weak magnetic anisotropy favors the appearance of localized resonances, contributing to MI variation starting at lower frequency ranges. At high frequencies, as shown in Fig. 4, similar MI behavior is noticed irrespective of the cut direction of the multilayered films, characterized by a double peak structure. This behavior reflects the quasi-static magnetic behavior, where the EA and HA cuts present similar magnetic features. Thus, in this system in which a weak magnetic anisotropy is induced, the MI variations can be devoted to the skin and FMR effect for the whole studied frequency range, leading to considerable MI changes even from hundreds of $\mathrm{MHz}$.

On the other hand, for a system with strong induced magnetic anisotropy, the dynamic magnetic behavior and MI changes are completely different than that described before, being dependent on the cut direction. For the EA cut of the $\mathrm{Py} / \mathrm{Ag}$ multilayered films, a single peak structure is verified for the whole frequency range, a consequence of the alignment of the easy magnetization axis with the $a c$ current and $d c$ magnetic field applied during the MI measurement. For 
this case, in the employed range of frequency, the skin effect is the unique mechanism that commands the MI variations. On the other hand, for the HA cut of the Py/Ag, the orientation among the easy magnetization axis, current, and magnetic field leads the MI to a double peak behavior. Up to $\sim 1.2 \mathrm{GHz}$, the position of the peaks remains unchanged at the $H_{s}$ field, indicating that the skin effect governs the magnetization dynamics. For frequencies above this value, besides the skin effect, the FMR effect also becomes an important mechanism responsible for variations in MI effect, a fact evidenced through the change of the peak's position in the magnetic field as the frequency is increased.

\section{Magnetoimpedance ratio}

Figure 6 shows the $\mathrm{MI}_{\max }$ vs. $f$ behavior obtained from the MI measurements for the multilayered films, considering the EA and HA cuts. As an important issue, the effective magnetic anisotropy directly influences the MI ratio. As seen in Figs. 6(a)-6(c), for the $\mathrm{Py} / \mathrm{Cu}$ multilayered films, the shape of the curves is similar, with the highest $\mathrm{MI}_{\text {max }}$ values at $\sim 1.5 \mathrm{GHz}$ for the $\mathrm{PyCu}_{15}$ and $\mathrm{PyCu}_{50}$ samples, irrespective of the cut direction, and at $\sim 0.6 \mathrm{GHz}$ and $\sim 1.0 \mathrm{GHz}$ for the $\mathrm{PyCu}_{100}$ in the EA and $\mathrm{HA}$ cuts. The $\mathrm{MI}_{\text {max }}$ values increase with the number of bilayers, reaching to $\sim 25 \%$, $\sim 100 \%$, and $\sim 250 \%$ for the $\mathrm{PyCu}_{15}, \mathrm{PyCu}_{50}$, and $\mathrm{PyCu}_{100}$ samples, respectively. In particular, the surprisingly discrepancy between the results for the $\mathrm{PyCu}_{100}$ film is still a question in debate.

For the Py/Ag multilayered films, the $\mathrm{MI}_{\max }$ vs. $f$ results are strongly dependent on the cut direction. For the HA cuts, the shape of the curves is similar to one observed for the $\mathrm{Py} / \mathrm{Cu}$ multilayered films. The $\mathrm{MI}_{\max }$ values seem to be at the same position in frequency, when compared with the $\mathrm{Py} / \mathrm{Cu}$ films, indicating a possible relation between the frequency where the maximum is observed with geometric parameters, such as number of bilayers, ratio of thicknesses $\mathrm{t}_{P y} / \mathrm{t}_{N M}$, and dimensions of the cut samples. In this case, the $\mathrm{MI}_{\text {max }}$ values reach to $\sim 6 \%, \sim 50 \%$, and $\sim 125 \%$ for the $\mathrm{PyAg}_{15}, \mathrm{PyAg}_{50}$, and PyAg 100 samples, respectively. On the other hand, for the EA cut, the $\mathrm{MI}_{\max }$ values become relevant just at high frequencies, above $\sim 1.0 \mathrm{GHz}$, reaching $\sim 14 \%$, $\sim 45 \%$, and $\sim 230 \%$ for the $\operatorname{PyAg}_{15}, \mathrm{PyAg}_{50}$, and $\mathrm{PyAg}_{100}$ multilayered films.

Thus, as consequence, it is possible to notice that for measurements performed with the HA cut of the multilayered films, the overlap between strong skin effect and the FMR, the main mechanisms responsible for the MI changes, leads to high values of $\mathrm{MI}_{\max }$ for a wide frequency range. However, when considered, the EA cut for both sets of films, the framework qualifies samples with distinct magnetic anisotropies for different technological applications. In this case, while a sample with weak magnetic anisotropy provides considerable impedance variations in a wide frequency range, similar to the ones obtained for the HA cut, a sample with well defined magnetic anisotropy presents the highest $\mathrm{MI}_{\text {max }}$ values in a narrow frequency band, due to the strong skin effect.

\section{Multiple peak behavior and numerical calculation}

Our results raise an interesting question about the structure of the peaks in the magnetoimpedance effect. In particular, here, is it verified a striking multiple peak structure at high frequencies for the HA cut of the Py/Ag multilayered films.

Usually, the induced magnetic anisotropy and its alignment with the $a c$ and $d c$ magnetic fields in a MI measurement favor the appearance of one single peak or a double peak structure, as frequently verified in several studies. ${ }^{25,32,33,37,44}$
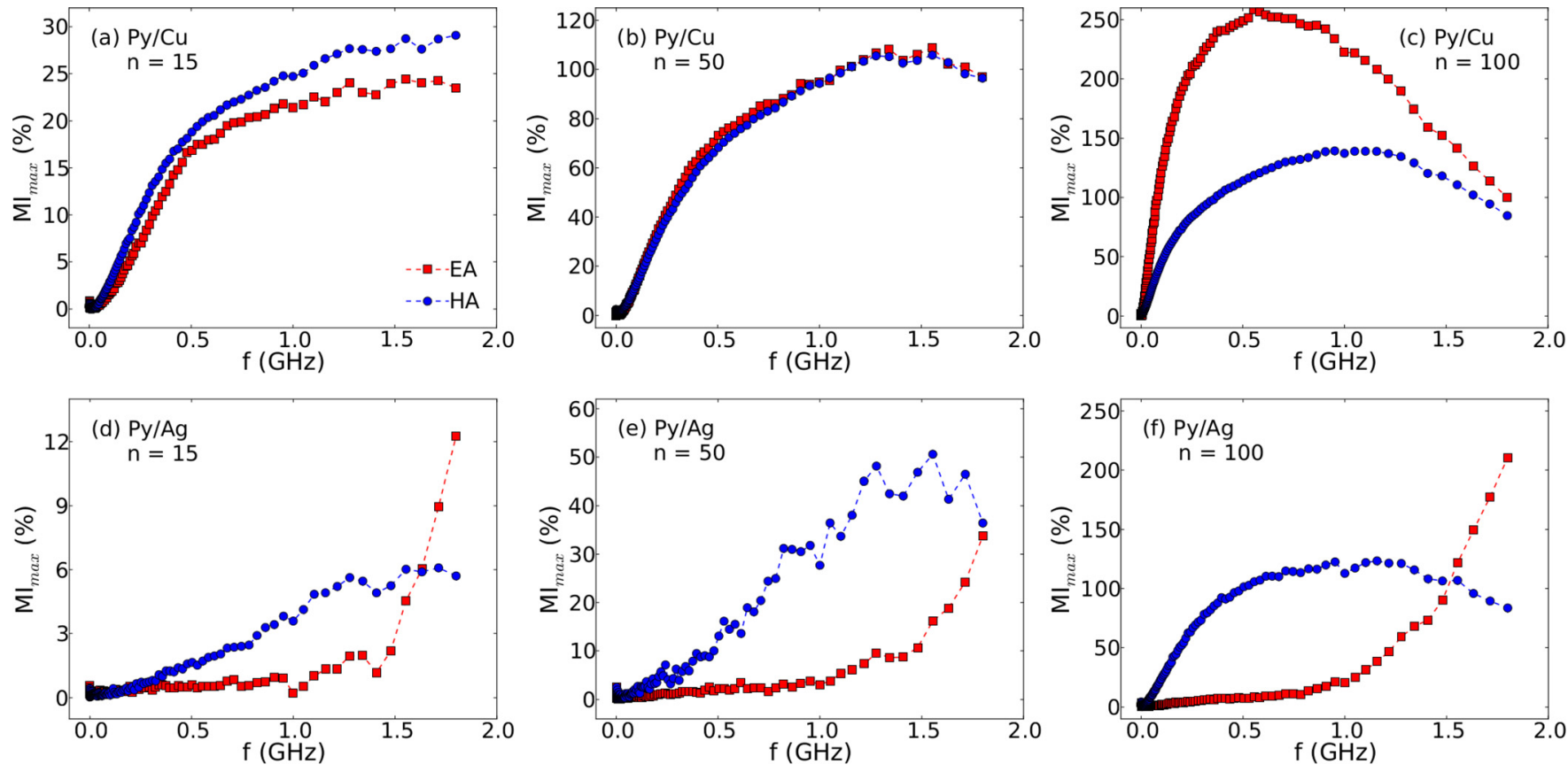

FIG. 6. $\mathrm{MI}_{\max }$ vs. $f$ for the Py/Cu and Py/Ag set of multilayered films obtained for the EA and HA cuts. Notice the striking between the shape of the curves when obtained for systems with weak and strong induced magnetic anisotropies. 

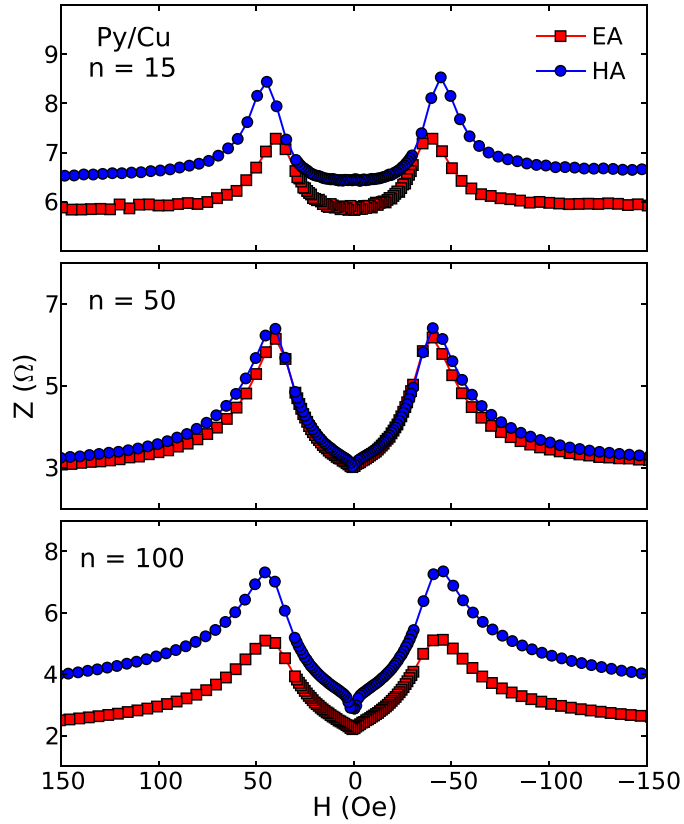

FIG. 7. Impedance $Z$ as a function of the magnetic field $H$, at $1.8 \mathrm{GHz}$, for the Py/Cu multilayered films, obtained for the EA and HA cuts.

In the case of the $\mathrm{Py} / \mathrm{Cu}$ multilayered films, the double peak structure is verified at high frequencies, irrespective of the alignment, as a consequence of the weak induced magnetic anisotropy. This behavior is already presented in Fig. 4 and is evidenced in Fig. 7. In the case of the Py/Ag multilayered films, a single peak structure is confirmed when the measurement is performed with the EA cut, also already presented in Fig. 5 and confirmed in Fig. 8, as expected.

However, when the MI effect is acquired for the Py/Ag multilayered films with the HA cut, at high frequencies, a noticeable multiple peak structure can be clearly verified, as presented in Fig. 8. At a first moment, due to the structure of

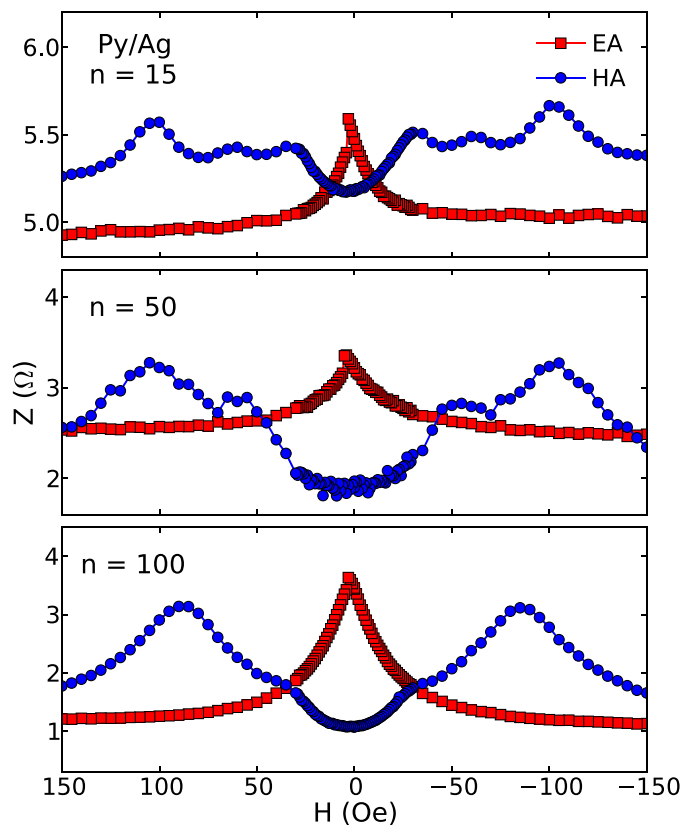

FIG. 8. Impedance $Z$ as a function of the magnetic field $H$, at $1.8 \mathrm{GHz}$, for the Py/Ag multilayered films, obtained for the EA and HA cuts. the samples, one can figure the multiple peak structure is devoted to different anisotropies with respect to the inner part of the each layer or pinning effects originated at the thin borders, leading different parts of the multilayered structure behave distinctly. However, this feature is associated with the very strong and well defined induced magnetic anisotropy verified in the Py/Ag multilayered films, with the exact orthogonality between the external magnetic field and uniaxial magnetic anisotropy induced during the growth of the multilayered films, as well as with the appearance of the FMR effect. This interpretation is supported by numerical calculation.

The influence on the MI effect of the induced magnetic anisotropy intensity and the alignment of the magnetic fields with respect to the magnetic anisotropy direction can be theoretically analyzed by using a approach previously reported by our group. ${ }^{45}$ To this end, a macrospin modified StonerWohlfarth model is considered to describe a general system with low electrical resistance and anisotropic magnetic properties, and calculate the static and dynamical magnetic properties. By taking together the magnetic susceptibility model proposed by Spinu et al. ${ }^{46}$ and the approach used to determine the magnetoimpedance behavior in a multilayered films systems reported by Panina et al., ${ }^{47}$ the results can provide information on the magnetic properties of a multilayer system, such as static magnetization curves, magnetic permeability, magnetoimpedance, as well as dispersion relation curves. By considering this approach, numerical calculation is performed extending the frequency range up to values in which is known that the FMR effect acts on the system.

In this case, the expression must present contributions from the ferromagnetic layers, the energy density is given by

$$
\begin{aligned}
E= & -\sum_{i=1}^{n} \vec{H} \cdot \vec{M}_{i}-\sum_{i=1}^{n}\left(H_{k i} / 2 M_{S i}\right)\left(\vec{M}_{i} \cdot \hat{u}_{k i}\right)^{2} \\
& +\sum_{i=1}^{n} 4 \pi M_{S i}^{2}\left(\hat{M}_{i} \cdot \hat{n}\right),
\end{aligned}
$$

where $n=15$, 50, or 100 bilayers, the first term is the Zeeman interaction, the second term describes the uniaxial magnetic anisotropy, and the third corresponds to demagnetizing energy term for a planar system, such as a thin film. In the terms, $\vec{M}_{i}$ and $M_{S i}$ are the magnetization vector and saturation magnetization value for each ferromagnetic layer, respectively. Besides, $H_{k i}=2 K_{u i} / M_{S i}$ is the anisotropy field for each layer, where $K_{u}$ is the uniaxial anisotropy constant, directed along the versor $\hat{u}_{k}$, with $\hat{u}_{k 1}=\hat{u}_{k 2}=\ldots=\hat{u}_{k n}$ $=\hat{u}_{k}$, as well as $M_{S 1}=M_{S 2}=\ldots=M_{S n}=M_{S}$, since there are similar ferromagnetic layers in the sample. The employed vectors and angles are depicted in Fig. 9. For further information on the theoretical approach and numerical calculation, we suggest Ref. 45.

From this point, the magnetic permeability and the impedance calculations require that some important parameters must be fixed, such as the gyromagnetic ratio $\gamma$, the phenomenological damping constant $\alpha$, thicknesses of the metallic and ferromagnetic layers, $t_{N M}$ and $t_{P y}$, and the conductivity of the metallic and ferromagnetic layers, $\sigma_{1}$ and $\sigma_{2}$, respectively. 


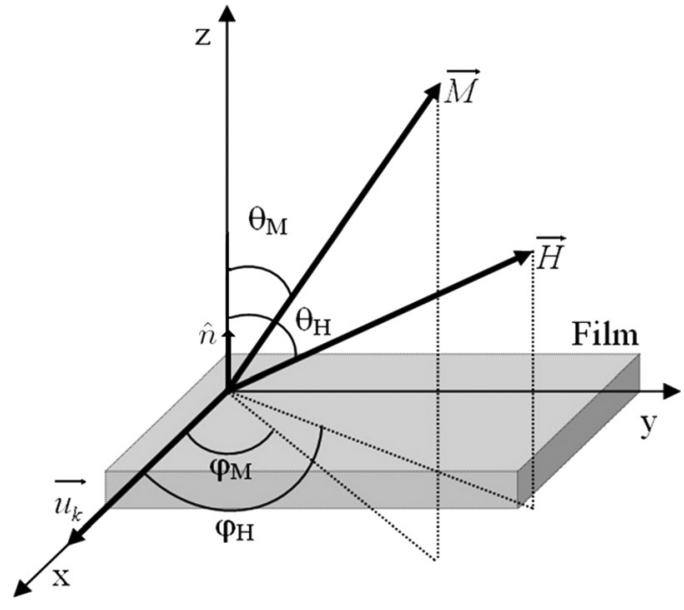

FIG. 9. Schematic diagram of the theoretical system and definitions of magnetization and magnetic field vectors considered for the numerical calculation of magnetization, magnetic permeability, and magnetoimpedance curves. In this case, $\vec{H}$ is the external magnetic field vector, $\theta_{H}$ and $\varphi_{H}$ are the angles that define orientation of the field vector, $\vec{M}$ is the magnetization vector, $\theta_{M}$ and $\varphi_{M}$ are the equilibrium angles of magnetization for a given magnetic field value, $\hat{u}_{k}$ is the unit vector along the uniaxial anisotropy direction, and $\hat{n}$ is a unit vector normal to the film plane.

In this study, the numerical calculations are performed using $\gamma=2.9 \times 10^{6} \mathrm{~Hz} / \mathrm{Oe}, \quad \alpha=0.0018, \quad t_{P y}=10 \mathrm{~nm}$, $t_{N M}=2.5 \mathrm{~nm}, \quad \sigma_{1}=6 \times 10^{7} \quad(\Omega \mathrm{m})^{-1}, \quad \sigma_{2}=\sigma_{1} / 4, \quad$ and $M_{s}=780 \mathrm{emu} / \mathrm{cm}^{3}$. Besides, to model a general ferromagnetic film, $\theta_{k}=90^{\circ}$ and $\varphi_{k}=0^{\circ}$ or $\varphi_{k} \sim 0^{\circ}$, while for the calculation, $\theta_{H}=90^{\circ}$ and $\varphi_{H}$ may vary between $0^{\circ}$ and $90^{\circ}$. In particular, all the $Z$ results are calculated for $f=1.8 \mathrm{GHz}$, frequency value in which the MI variations and $Z$ peaks are induced mainly by the FMR effect, when $\theta_{H}=90^{\circ}$ and $\varphi_{H} \sim 90^{\circ}$. Note that the results are obtained for a general system with anisotropic magnetic properties using the Stoner-Wolfart modified model, and, for this reason, they do not allow a direct comparison between the calculated $Z$ and the presented experimental results.

Figure 10 shows the numerical calculation for the $Z$ vs. $H$ for different values of anisotropy field $H_{k}$, i.e., uniaxial anisotropy constants or induced magnetic anisotropy intensity, for $\theta_{k}=90^{\circ}, \varphi_{k}=0^{\circ}, \theta_{H}=90^{\circ}$, and $\varphi_{H}=90^{\circ}$ or, in the

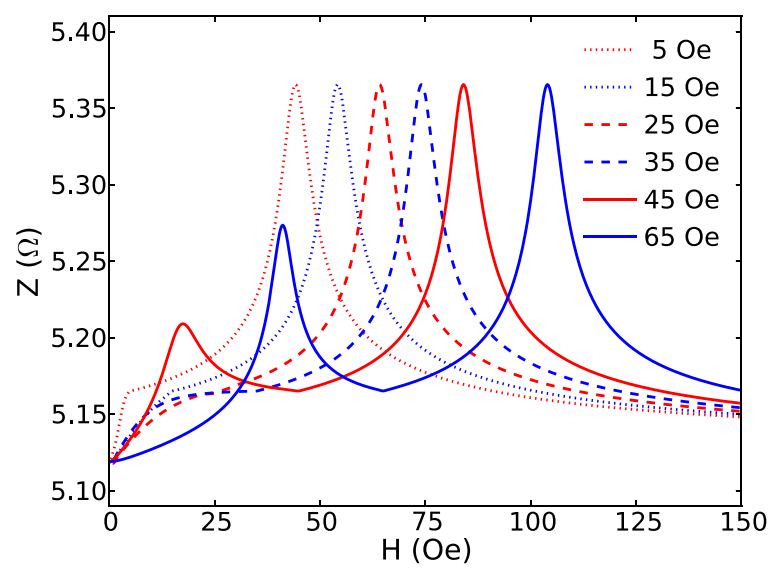

FIG. 10. Numerical calculation for the impedance $Z$ vs. external magnetic field $H$, at $1.8 \mathrm{GHz}$, for distinct anisotropy fields $H_{k}$. Here, $\theta_{k}=90^{\circ}$, $\varphi_{k}=0^{\circ}, \theta_{H}=90^{\circ}$, and $\varphi_{H}=90^{\circ}$. other words, when the $d c$ magnetic field is applied along the hard magnetization axis. The curves are presented only for positive magnetic field values, although this behavior is also observed for negative field values. So, from the figure, it is possible to notice a change of the $Z$ behavior, from a traditional double peak to a multiple peak structure as the anisotropy field/intensity is increased. This evolution of the peaks and the multiple peak structure are not frequently observed since the condition of strong and very well defined magnetic anisotropy is hard to be reached. Thus, keeping in mind the dependence of the MI peak structure with the magnetic anisotropy, it becomes reasonable the double peak structure verified for the weak anisotropic $\mathrm{PyCu}_{15}, \mathrm{PyCu}_{50}$, and $\mathrm{PyCu}_{100}$ multilayered films (Fig. 7), as well as the multiple peak structure for strong anisotropic $\mathrm{PyAg}_{15}$ and $\mathrm{PyAg}_{50}$ ones (Fig. 8).

Next, keeping $\theta_{H}=90^{\circ}, H_{k}=65 \mathrm{Oe}, \theta_{H}=90^{\circ}$, and $\varphi_{H}=90^{\circ}$, Fig. 11 shows the numerical calculation for the $Z$ vs. $H$ for different $\varphi_{k}$ values. In this case, the increase in $\varphi_{k}$ models the stored stress leading to small deviations of the anisotropy in the sample. For an exact alignment between the $d c$ magnetic field and the hard magnetization axis, and a well defined magnetic anisotropy, $\varphi_{k}=0^{\circ}$, the $Z$ result is a multiple peak structure. As the anisotropy deviation is raised, depicted by larger values of $\varphi_{k}$, the raise of anisotropy deviation and the lack of orthogonality between $d c$ magnetic field and easy magnetic anisotropy direction suppress the multiple peak structure, leading to a double peak one. This evolution of the $Z$ behavior is clearly verified for the set of Py/Ag multilayered films, as the number of bilayers $n$ is increased. In this case, as presented in Fig. 8, the multiple peak structure is observed for the $\mathrm{PyAg}_{15}$ and $\mathrm{PyAg}_{50}$ multilayered films, and the double peak structure is seen for the $\mathrm{PyAg}_{100}$ ones, an evolution devoted to the stress stored in the film as the thickness increases and to a small raise of the magnetic anisotropy deviation.

Thus, once a straight relation of the MI effect with induced magnetic anisotropy intensity and the alignment of the magnetic fields with respect to the magnetic anisotropy direction are identified, the origin the $Z$ peak structures can be elucidated using the classic relationship between the

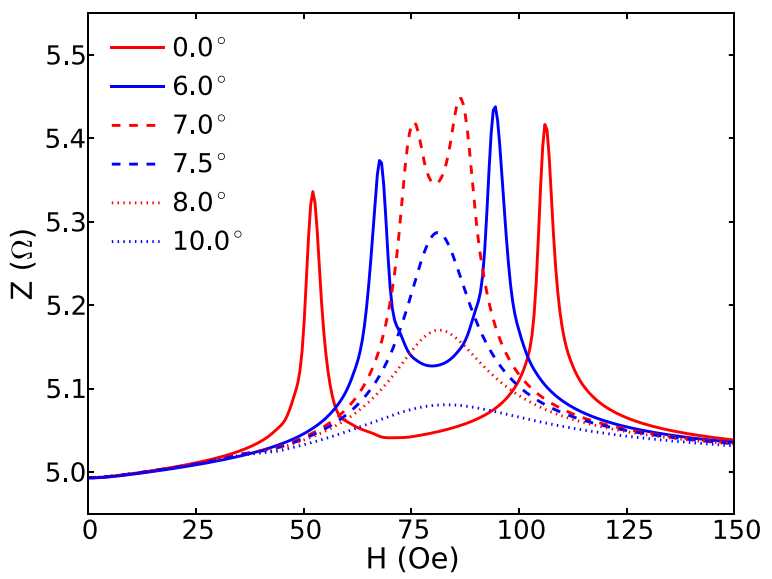

FIG. 11. Numerical calculation for the impedance $Z$ vs. external magnetic field $H$, at $1.8 \mathrm{GHz}$, for different values of the angle of magnetic anisotropy direction $\varphi_{k}$. Here, $\theta_{k}=90^{\circ}, H_{k}=65$ Oe, $\theta_{H}=90^{\circ}$, and $\varphi_{H}=90^{\circ}$. 
resonance frequency and the external magnetic field. Figure 12 presents the numerical calculation of the dispersion relation in systems with weak and strong anisotropy for $\varphi_{H}=0^{\circ}$ and $90^{\circ}$. For these calculations, $\theta_{k}=90^{\circ}, \varphi_{k}=0^{\circ}, \theta_{H}=90^{\circ}$, $H_{k}=5 \mathrm{Oe}$ and $H_{k}=65$ Oe for systems with weak and strong anisotropy, respectively, and $\varphi_{H}=0^{\circ}$ and $90^{\circ}$.

It is well known that for isotropic systems (not shown here), the dispersion relations are the same, irrespective the orientation of the magnetic field. Considering anisotropic systems, the shape of the curves is similar for each orientation between magnetic field and magnetic anisotropy direction, irrespective of the anisotropy intensity. For $\varphi_{H}=0^{\circ}$, when the magnetic field is along the easy magnetization axis, just on resonance positive field is verified for a given resonance frequency $f_{r}$, leading to a double peak structure in the $Z$ curves.

However, the main fingerprint that reflects the magnetic properties of the considered systems, such as magnetic anisotropies and coupling, is verified when $\varphi_{H}=90^{\circ}$, i.e., the magnetic field is along the hard magnetization axis of the theoretical sample. In this case, it is related to the resonance frequency and magnetic field ranges in which the dispersion relation is not an univocal function, indicated in the figure by the gray regions. For a system with strong anisotropy, there is a wide range where two positive magnetic field values satisfy the resonance condition, leading to a multiple peak structure in the $Z$ curves. In principle, similar multiple peak structure could be verified experimentally for systems with weak anisotropy, however, it is not usually obtained because
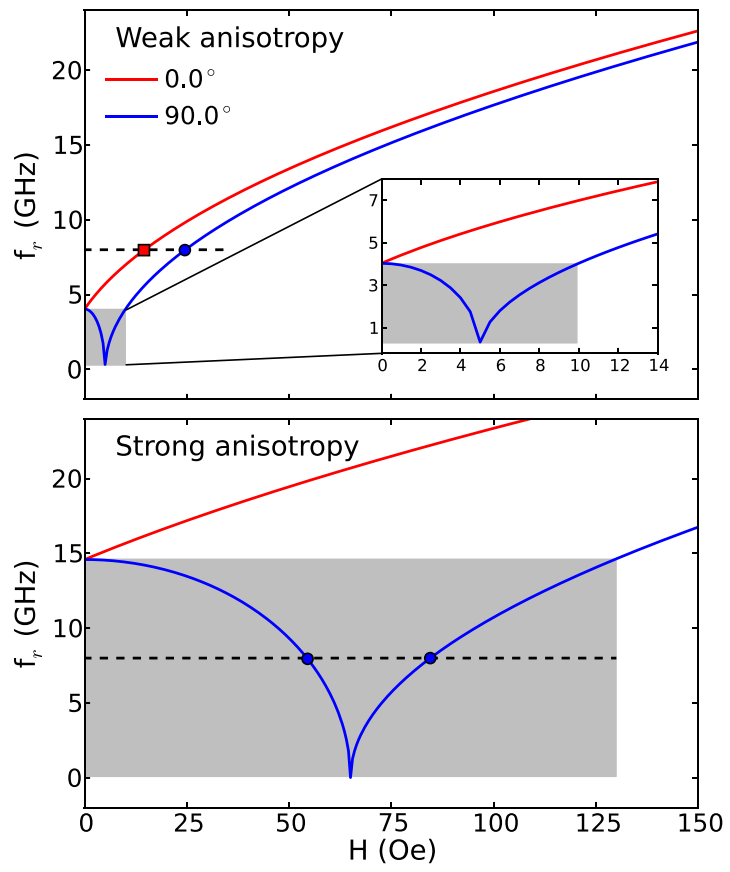

FIG. 12. Numerical calculation of the dispersion relation for systems with weak and strong anisotropy obtained for $\varphi_{H}=0^{\circ}$ and $90^{\circ}$, i.e., $\varphi_{H}$ being the angle between the external magnetic field and magnetic anisotropy directions. Note that these considered theoretical situations are equivalent to the experimental EA and HA cuts, respectively. Here, $\theta_{k}=90^{\circ}, \varphi_{k}=0^{\circ}$, $\theta_{H}=90^{\circ}$, while $H_{k}=5 \mathrm{Oe}$ and $H_{k}=65 \mathrm{Oe}$ for systems with weak and strong anisotropy, respectively. Each crossing of the dashed line with the dispersion relation indicates the existence of a peak in the $Z$ curve. Inset: Detailed view of the dispersion relation at low fields for systems with weak anisotropy. of the overlap of the peaks, due to the restricted magnetic field range in which they are observed.

The theoretical situations of $\varphi_{H}=0^{\circ}$ and $90^{\circ}$ are equivalent to the experimental EA and HA cuts. Consequently, through this point of view, the dispersion relation directly provides interpretation for the $Z$ curves measured for the multilayered films. For the Py/Cu ones with weak anisotropy in both cuts, a double peak structure in the $Z$ curves is confirmed through just one resonant condition for positive fields in the dispersion relation or overlap of the $Z$ peaks. In particular, we are not able to solve a multiple peak structure due to the limited frequency range of the equipment and magnetic field resolution. Notice in the inset of the figure that at $f=1.8 \mathrm{GHz}$, the double peak structure would appear between $\sim 4$ and $\sim 6$ Oe.

For the Py/Ag multilayered films in the EA cut, double peak structure in the $Z$ curves is verified, as expected. On the other hand, the $Z$ curves for the HA cut, multiple peak structure are confirmed for the samples with 15 and 50 bilayers, in concordance with the dispersion relation obtained for systems with strong anisotropy. Note that by producing samples with strong and well defined anisotropy, it is possible to split the resonance magnetic fields for a given frequency that makes possible the experimental observation of the multiple peak structure. For the film with 100 bilayers, a double peak structure is obtained possibly due to a very small misalignment of the anisotropy direction with respect to the magnetic field, a fact that leads to considerable raise of the resonance frequencies close to the anisotropy field in the dispersion relation, above the upper frequency limit of the measurement equipment.

Clearly, the magnetic anisotropy has a fundamental role in the magnetoimpedance effect behavior for both spacer materials. By playing with the nature of the $\mathrm{Cu}$ and $\mathrm{Ag}$ nonmagnetic metallic spacers, the multilayered films can be used in sensor applications and, both the frequency and field response can be tailored to fulfill the requirements of a given device.

\section{CONCLUSION}

In summary, in this paper, we performed a systematic experimental study of the high-frequency response of magnetization dynamics through magnetoimpedance effect in Permalloy-based multilayered thin films produced with two different non-magnetic metallic spacers: $\mathrm{Cu}$ and Ag. By employing these spacers, it was possible to produce samples with completely different magnetic behaviors. As expected, while the multilayered films with $\mathrm{Cu}$ had weak induced magnetic anisotropy, the ones with Ag presented strong magnetic anisotropy, as well as a well defined anisotropy direction.

The magnetic properties were reflected on the dynamic magnetic behavior. For the $\mathrm{Py} / \mathrm{Cu}$ multilayered films, the peak's configuration evolves from a single, at low frequencies, to a double peak structure at high frequencies, irrespective of the cut direction. Thus, in this system in which a weak magnetic anisotropy is induced, the MI variations can be devoted to the skin and FMR effect for the whole studied frequency range, leading to considerable $\mathrm{MI}$ changes even 
from hundreds of $\mathrm{MHz}$, reaching to $\sim 25 \%, \sim 100 \%$, and $\sim 250 \%$ for the $\mathrm{PyCu}_{15}, \mathrm{PyCu}_{50}$, and $\mathrm{PyCu}_{100}$ samples, respectively.

For the Py/Ag multilayered films, the dynamic magnetic behavior and MI changes are completely different than that described before, being dependent on the cut direction. For the EA cut, a single peak structure is verified for the whole frequency range, in which the skin effect is the unique mechanism that commands the MI variations. In this case, the $\mathrm{MI}_{\max }$ values become relevant just at high frequencies, above $\sim 1.0 \mathrm{GHz}$, reaching $\sim 14 \%, \sim 45 \%$, and $\sim 230 \%$ for the $\mathrm{PyAg}_{15}, \mathrm{PyAg}_{50}$, and PyAg 100 multilayered films. For the $\mathrm{HA}$ cut of the Py/Ag, the observed double peak behavior is associated with skin and FMR effects. The shape of the $\mathrm{MI}_{\max }$ curves is similar to one observed for the $\mathrm{Py} / \mathrm{Cu}$ multilayered films, and the $\mathrm{MI}_{\max }$ values reach to $\sim 6 \%, \sim 50 \%$, and $\sim 125 \%$ for the $\operatorname{PyAg}_{15}, \operatorname{PyAg}_{50}$, and $\mathrm{PyAg}_{100}$ samples, respectively. Thus, we confirm that these features are very important for the use of multilayered films in sensor applications and, both the frequency and field response can be tailored to fulfill the requirements of a given device.

As a final and very important remark, our results raised an interesting question about the structure of the peaks in the magnetoimpedance effect. To interpret them, we considered two single models together, the transverse magnetic susceptibility model proposed by Spinu et al. ${ }^{46}$ and the approach to magnetoimpedance behavior reported by Panina et al. ${ }^{47}$ and performed numerical calculation modeling the effect of weak/strong magnetic anisotropy on the MI response. Thus, we verified the influence on the MI effect of the induced magnetic anisotropy intensity and the alignment of the magnetic fields with respect to the magnetic anisotropy direction. Based on this procedure, we supported the interpretation that observed MI effect curves, in particular, the multiple peak structures, are a result of the overlap of contributions from the magnetic anisotropy and the orthogonality of the hard magnetization axis with the $d c$ magnetic field at high frequencies, where the FMR effect is present.

\section{ACKNOWLEDGMENTS}

The research is partially supported by the Brazilian agencies CNPq (Grants Nos. 471302/2013-9, 310761/20115, 476429/2010-2, 555620/2010-7, 307951/2009-0, and 482735/2009-0), CAPES, FAPERJ (Nos. E-26/102.943/2008 and E-26/112.697/2012), and FAPERN (Grant PPP No. 013/2009 and Pronem No. 03/2012). M.A.C. and F.B. acknowledge financial support of the INCT of Space Studies.

${ }^{1}$ T. Gerrits, H. van den Berg, J. Hohlfeld, L. Bar, and T. Rasing, Nature 418, 509 (2002).

${ }^{2}$ C. Boone et al., Phys. Rev. B 79, 140404 (2009).

${ }^{3}$ K. V. Thadani et al., Phys. Rev. B 78, 024409 (2008).
${ }^{4}$ P. Delooze, L. Panina, D. Mapps, K. Ueno, and H. Sano, J. Magn. Magn. Mater. 272-276(Part 3), 2266 (2004).

${ }^{5}$ M. Knobel and K. R. Pirota, J. Magn. Magn. Mater. 242-245(Part 1), 33 (2002).

${ }^{6}$ L. Kraus, J. Magn. Magn. Mater. 195, 764 (1999).

${ }^{7}$ M. Vázquez, J. Magn. Magn. Mater. 226-230(Part 1), 693 (2001).

${ }^{8}$ M. A. Corrêa et al., Appl. Phys. Express 6, 093001 (2013).

${ }^{9}$ M. Carara, A. Gundel, M. N. Baibich, and R. L. Sommer, J. Appl. Phys 84, 3792 (1998).

${ }^{10}$ R. S. Beach and A. E. Berkowitz, Appl. Phys. Lett. 64, 3652 (1994).

${ }^{11}$ L. V. Panina and K. Mohri, Appl. Phys. Lett. 65, 1189 (1994).

${ }^{12}$ J. Velázquez, M. Vázquez, D.-X. Chen, and A. Hernando, Phys. Rev. B 50, 16737 (1994).

${ }^{13}$ K. Mohri, L. Panina, T. Uchiyama, K. Bushida, and M. Noda, IEEE Trans. Magn. 31, 1266 (1995).

${ }^{14}$ D. P. Makhnovskiy, L. V. Panina, and D. J. Mapps, Appl. Phys. Lett. 77, 121 (2000).

${ }^{15}$ M. Vazquez, M. Knobel, M. L. Sanchez, R. Valenzuela, and A. P. Zhukov, Sens. Actuators, A 59, 20 (1997).

${ }^{16}$ R. B. da Silva, A. M. H. de Andrade, A. M. Severino, L. F. Schelp, and R. L. Sommer, J. Appl. Phys. 91, 7436 (2002).

${ }^{17}$ M. Carara et al., J. Appl. Phys. 98, 033902 (2005).

${ }^{18}$ K. D. Sossmeier, F. Bohn, H. Chiriac, and M. Carara, J. Phys. D: Appl. Phys. 40, 3233 (2007).

${ }^{19}$ F. L. A. Machado, C. S. Martins, and S. M. Rezende, Phys. Rev. B 51, 3926 (1995).

${ }^{20}$ A. N. Medina, M. Knobel, S. Salem-Sugui, and F. G. Gandra, J. Appl. Phys. 79, 5462 (1996).

${ }^{21}$ R. L. Sommer and C. L. Chien, Phys. Rev. B 53, R5982 (1996).

${ }^{22}$ A. Santos et al., J. Appl. Phys. 79, 6546 (1996).

${ }^{23}$ K. R. Pirota, L. Kraus, M. Knobel, P. G. Pagliuso, and C. Rettori, Phys. Rev. B 60, 6685 (1999).

${ }^{24}$ F. Amalou and M. A. M. Gijs, J. Appl. Phys. 95, 1364 (2004).

${ }^{25}$ R. L. Sommer and C. L. Chien, Appl. Phys. Lett. 67, 3346 (1995).

${ }^{26}$ A. D. C. Viegas et al., J. Appl. Phys. 101, 033908 (2007).

${ }^{27}$ A. M. H. de Andrade et al., J. Magn. Magn. Mater. 272-276(Part 3), 1846 (2004).

${ }^{28}$ D. de Cos et al., Sens. Actuators, A 129, 256 (2006).

${ }^{29}$ M. Gamino et al., J. Magn. Magn. Mater. 320, e308 (2008).

${ }^{30}$ R. B. da Silva et al., Appl. Phys. Lett. 94, 042501 (2009).

${ }^{31}$ M. A. Corrêa et al., J. Phys. D: Appl. Phys. 43, 295004 (2010).

${ }^{32}$ M. S. Marques et al., Thin Solid Films 520, 2173 (2012).

${ }^{33}$ M. A. Corrêa et al., J. Magn. Magn. Mater. 320, e25 (2008).

${ }^{34}$ T. Morikawa et al., IEEE Trans. Magn. 32, 4965 (1996).

${ }^{35}$ G. Kurlyandskaya et al., J. Magn. Magn. Mater. 242-245, 291 (2002).

${ }^{36}$ G. V. Kurlyandskaya et al., J. Phys.: Condens. Matter 16, 6561 (2004).

${ }^{37}$ M. A. Corrêa et al., J. Phys. D: Appl. Phys. 41, 175003 (2008).

${ }^{38}$ M. A. Corrêa, A. D. C. Viegas, R. B. da Silva, A. M. H. de Andrade, and R. L. Sommer, Phys. B: Condens. Matter 384, 162 (2006).

${ }^{39}$ M. A. Corrêa, A. D. C. Viegas, R. B. da Silva, A. M. H. de Andrade, and R. L. Sommer, J. Appl. Phys. 101, 043905 (2007).

${ }^{40}$ H. Chiriac, M. Tibu, A.-E. Moga, and D. D. Herea, J. Magn. Magn. Mater. 293, 671 (2005).

${ }^{41}$ J. Devkota, A. Ruiz, P. Mukherjee, H. Srikanth, and M.-H. Phan, IEEE Trans. Magn. 49, 4060 (2013).

${ }^{42}$ S. Nazari Nejad, A. Fomani, and R. Mansour, IEEE Trans. Magn. 49, 3874 (2013)

${ }^{43}$ J. M. Barandiaran, A. Garcia-Arribas, and D. de Cos, J. Appl. Phys. 99, 103904 (2006).

${ }^{44}$ D. P. Makhnovskiy, N. Fry, L. V. Panina, and D. J. Mapps, J. Appl. Phys. 96, 2150 (2004).

${ }^{45}$ M. A. Corrêa et al., J. Appl. Phys. 110, 093914 (2011).

${ }^{46}$ L. Spinu, I. Dumitru, A. Stancu, and D. Cimpoesu, J. Magn. Magn. Mater. 296, 1 (2006).

${ }^{47}$ L. V. Panina, D. P. Makhnovskiy, D. J. Mapps, and D. S. Zarechnyuk, J. Appl. Phys. 89, 7221 (2001). 